\title{
曲線材を含む剖節骨組に對する一般化した撓危法
}

准目第尾健 三

\section{丽容 襣溉}

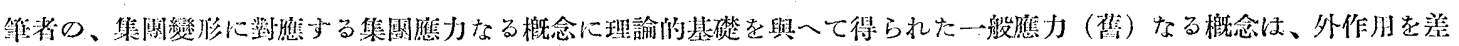

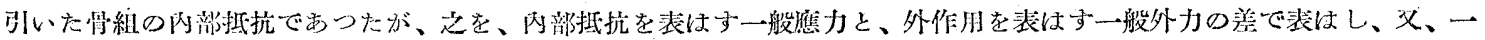

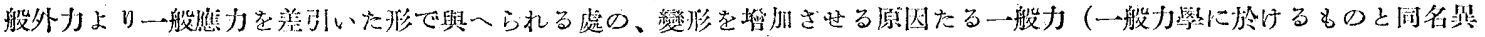

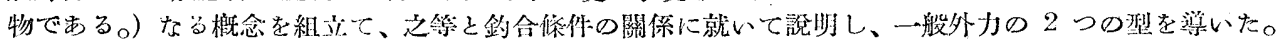

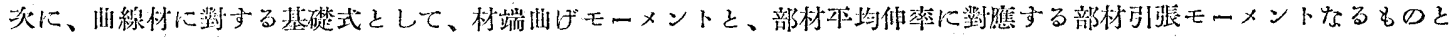

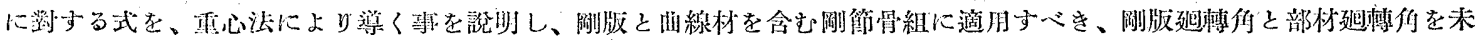

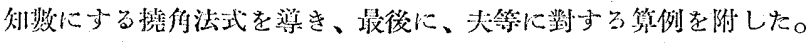

\section{目次}

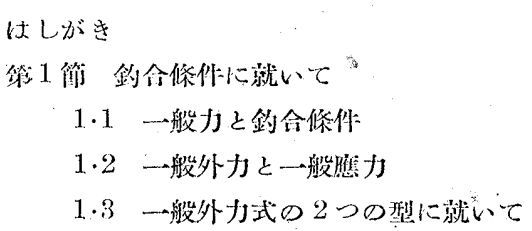

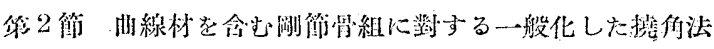

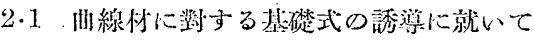

$2 \cdot 2$ 邀合條作

$2 \cdot 3$ 筧们沾式

2.4 管 例

\section{ばしがき}

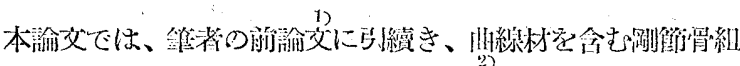

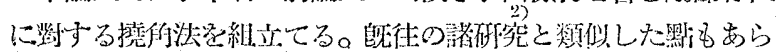

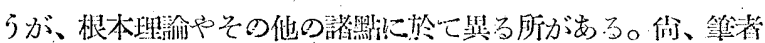

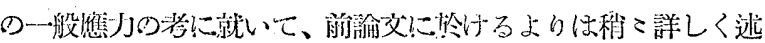

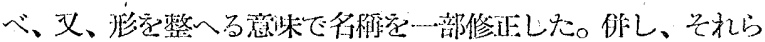

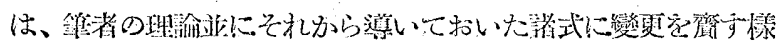
なものでない四は云ふまでもない。

\section{第|節 釣合條件に就いて}

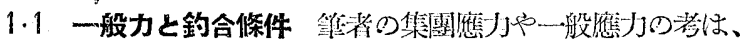

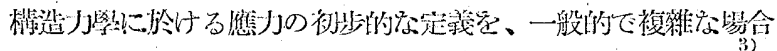

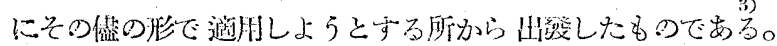

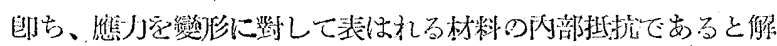

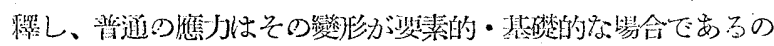

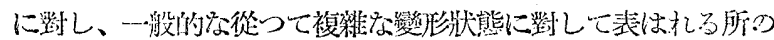

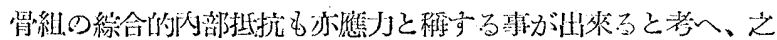

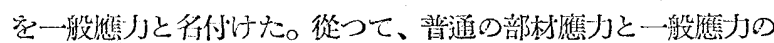

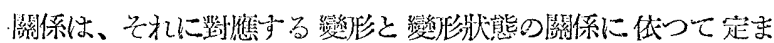

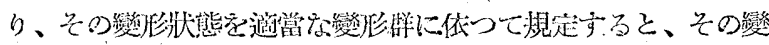

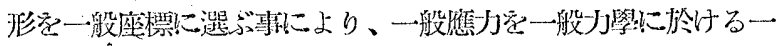

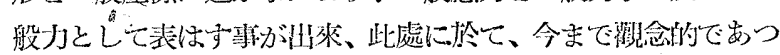

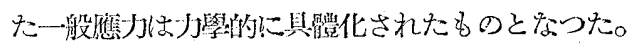

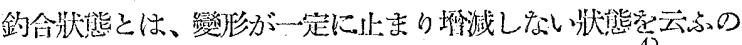

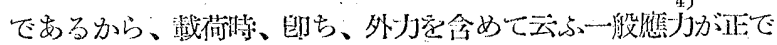

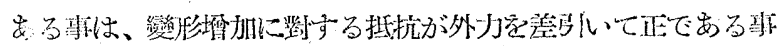

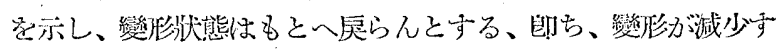

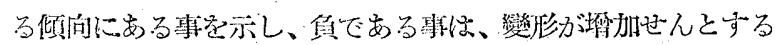

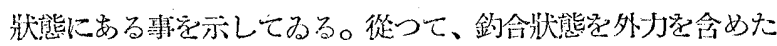

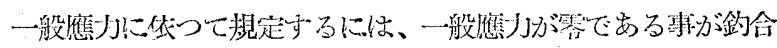

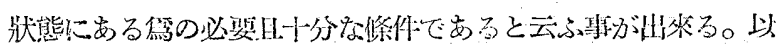

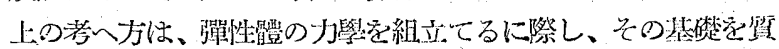

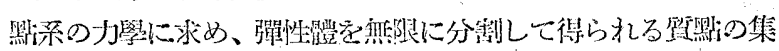

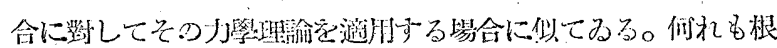

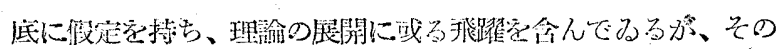

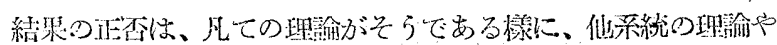

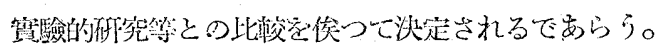

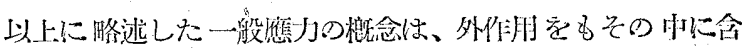

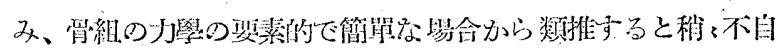

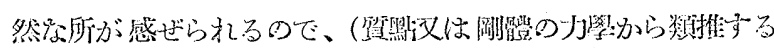

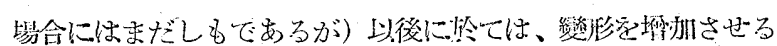

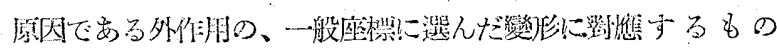

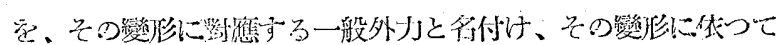

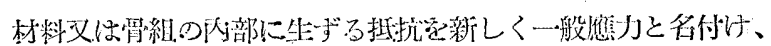

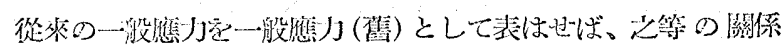
は、

$$
Z_{i}=S_{i}-P_{i}
$$

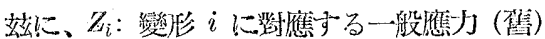

$$
\begin{aligned}
& \boldsymbol{S}_{i}: \quad " \quad, \quad \text { - 般應力 } \\
& \boldsymbol{P}_{i} \text { ! " " 一般外开 }
\end{aligned}
$$

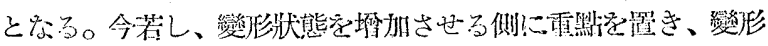

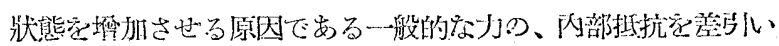

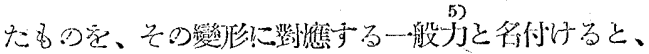

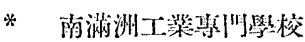

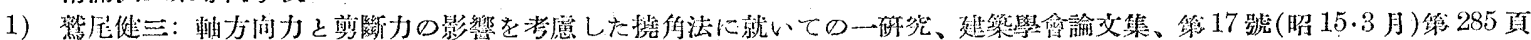

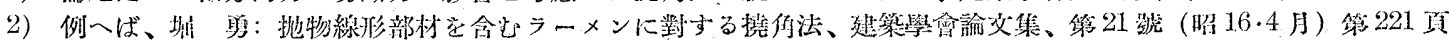

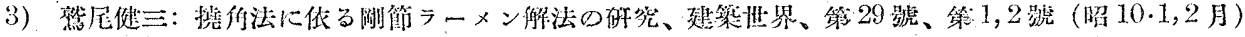

4) 脚流: 1) の文缺參照

5）此處に云ふ「一般力」は、一般力攀に於ける「一般力」と、刚容に於て数似點があるが、同じものではない。 


$$
Z_{i}=-Z_{i}=\boldsymbol{P}_{i}-S_{i}
$$

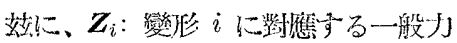

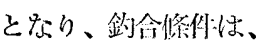

$$
\boldsymbol{Z}_{i}=0 \text { 又は } \boldsymbol{P}_{i}=\boldsymbol{S}_{i}
$$

として表はされる。此の釣合條件式の形は、一質點又は洞體の

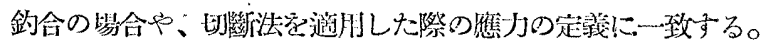

1 -2 一般外力と一般應力 岡版と彈性線材からなる骨組に

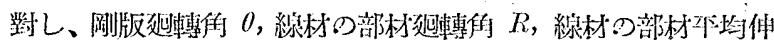

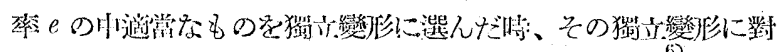

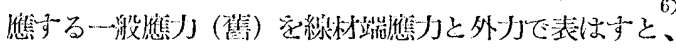

$$
\begin{aligned}
Z_{i} & =\sum_{A}\left\{\sum_{K} M_{A K}-\sum M_{A}^{0}\right\} \xi_{A(i)} \\
& -\sum_{A B}\left\{M_{A B}+M_{B A}+\sum M_{A B}^{0}\right\} \eta_{A B(i)} \\
& +\sum_{A B}\left\{N_{A B}-\sum P_{x}(1-x / l)\right\} l_{A B^{\odot}=A B(i)} \\
& -\sum P c_{(i)} \ldots \ldots \ldots \ldots \ldots \ldots \ldots \ldots \ldots \ldots \ldots \ldots \ldots \ldots \ldots
\end{aligned}
$$

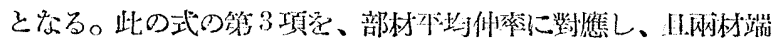

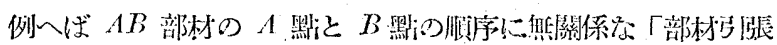
モーメント」とも究らべき、

$$
\begin{aligned}
T_{A B} & =\left\{N_{A B l_{A B}}-\sum P_{m}(l-x)\right\} \\
& =\left\{N_{B A} l_{A B}+\Sigma P_{m} x\right\} \ldots \ldots \ldots .
\end{aligned}
$$

で置き挨へると、一船水は、

$$
\begin{aligned}
\boldsymbol{Z}_{i} & =\sum_{A}\left\{\sum M_{A}^{0}-\sum_{K} M_{A K}\right\} \xi_{A(i)} \\
& +\sum_{A B}\left\{\sum M_{A B}^{0}+M_{A B}+M_{B A}\right\} \eta_{A B(i)} \\
& \left.-\sum_{A B} T_{A B^{\zeta}, A B(i)}+\sum P_{(i)}\right) \ldots \ldots \ldots \ldots
\end{aligned}
$$

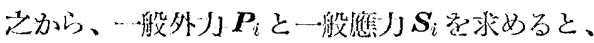

$$
\left.\begin{array}{rl}
\boldsymbol{P}_{i} & =\sum_{A}\left\{\sum M_{A}^{9}-\sum_{K} C_{A K}\right\} \xi_{A(i)} \\
& +\sum_{A B}\left\{\sum M_{A B}^{0}+C_{A B+}+C_{B A}\right\} \eta_{A B(i)}
\end{array}\right)
$$

$$
\begin{aligned}
& -\sum_{A B} D_{A B}^{\zeta}{ }_{A B(i)}+\sum P c_{(i)} \\
& \boldsymbol{S}_{i}=\sum_{A}\left\{\sum_{K} M_{A K(\theta i R e)}\right\} \xi_{A(i)}
\end{aligned}
$$

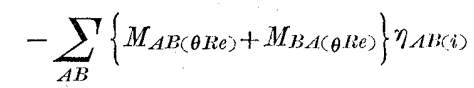

$$
\begin{aligned}
& +\sum_{A B} T_{A B(\theta R e)^{\zeta} A B(i)} \\
& \text { 赫に、 } M_{A B}=M_{A B(\theta R e)}+C_{A B} \\
& T_{A B}=T_{A B(\theta R e)}+D_{A B}
\end{aligned}
$$

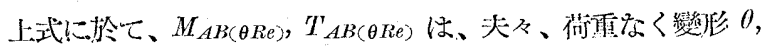

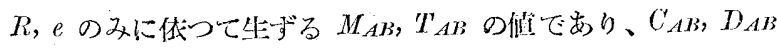

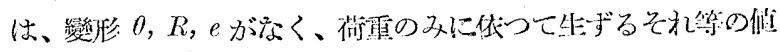
である。

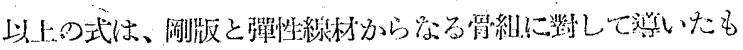

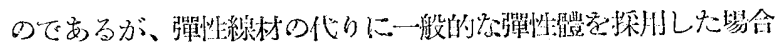

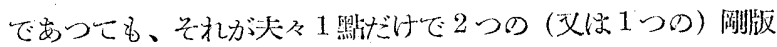

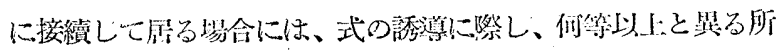

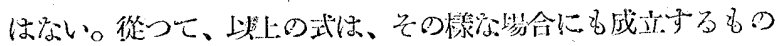

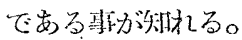

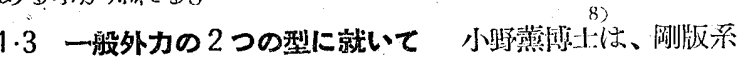

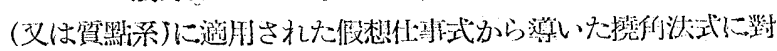

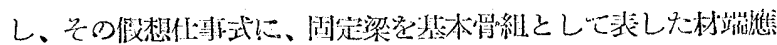

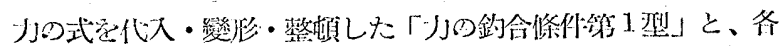

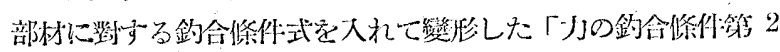

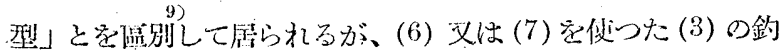

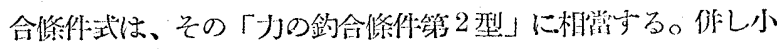

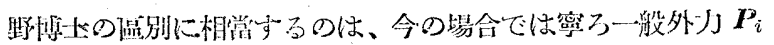

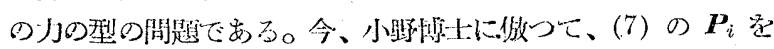

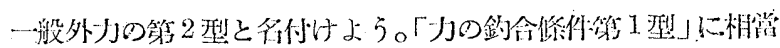

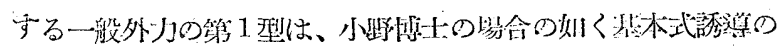

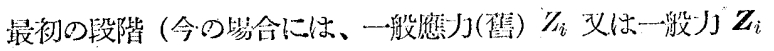

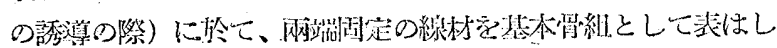

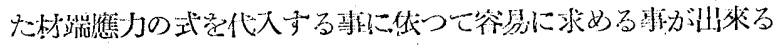

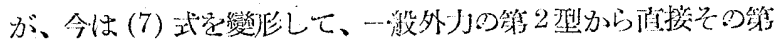

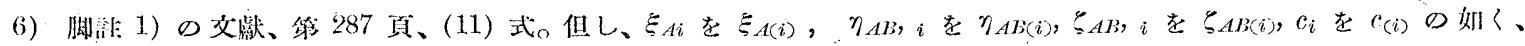

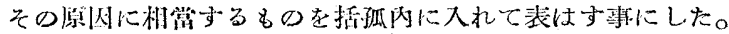

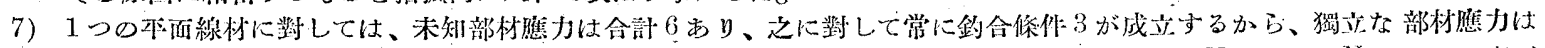

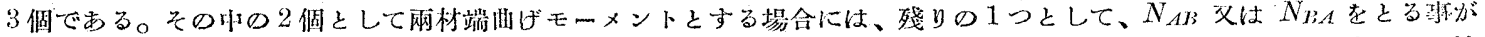

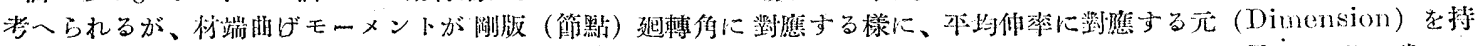

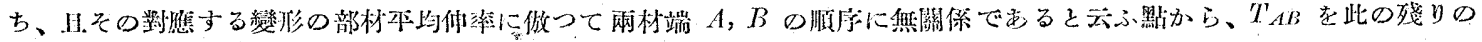
1つとして遙ぶのが、今の場合適常であると将へられる。

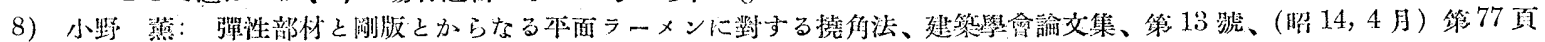

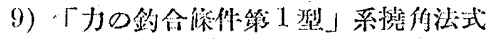

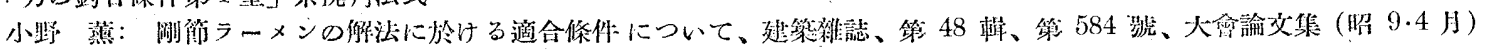
第 368 頁

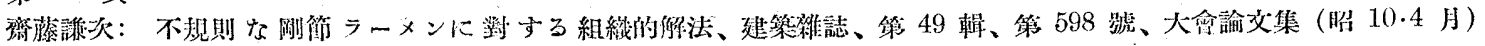
第 357 页

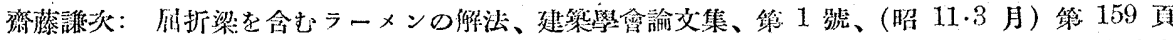

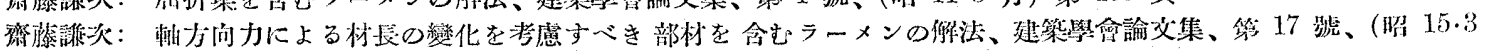
月) 被 295 面

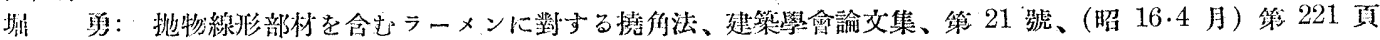

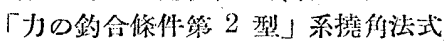

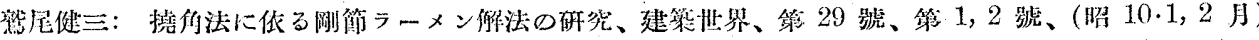

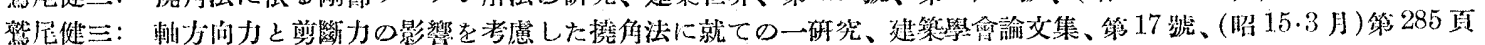


1 型空求めて見上5。

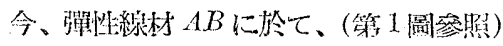

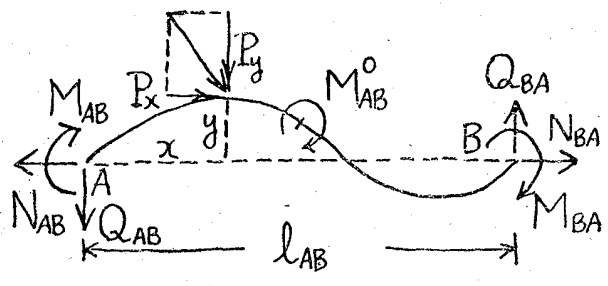

第 1 圖

$$
\left.\begin{array}{l}
Q_{A B}=Q_{A B(\theta R e)}+Q_{A B(P)} \\
N_{A B}=N_{A B(\theta R e)}+N_{A B(P)}
\end{array}\right\}
$$

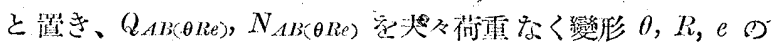

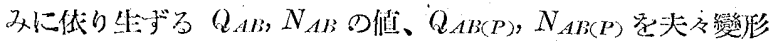

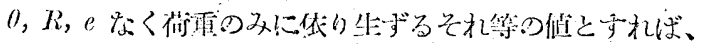

$$
\left.\begin{array}{rl}
Q_{A B(\theta R e)} & =Q_{B A(\theta R e)} \\
& =\left(M_{A B(\theta R e)}+M_{B A(\theta R e)}\right) / l_{A B} \\
N_{A B(\theta R e)} & =N_{B A(\theta R e)} / l_{A B}=T_{A B(\theta R e)} / l_{A B}
\end{array}\right\}
$$

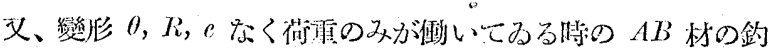
合條作より。

$$
\begin{aligned}
Q_{A B(P)} & =\left(\Sigma M_{A B}^{\prime}+C_{A B}^{\prime}+C_{B A}\right) / l_{A B} \\
& +\Sigma P_{m} y / l-\Sigma P y(1-x / l) \\
Q_{B A(P)} & =\left(\Sigma M_{A B}^{0}+C_{A B}+C_{B A}\right) / l_{A B} \\
& +\Sigma P_{x y} / l+\Sigma P_{y} x / l \\
N_{A B(P)} & =N_{B A(P)}+2 P_{x}
\end{aligned}
$$

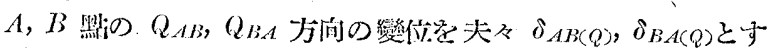
れば。

$\eta_{A B(i)}=-\left(\delta_{A B(Q)}+\delta_{B A(Q)}\right) / l_{A B}$

尔る故、(9)，(10)，(11) より、

$$
\begin{aligned}
& \left(\Sigma M_{A B}^{0}+C_{A B}+C_{B A}^{\prime}\right) \eta_{A B(i)}=-\left\{Q_{A B(P) \delta_{A B(Q)}}\right. \\
& \left.+Q_{B A(P)} \delta_{B A(Q)}\right\} \\
& -\Sigma P_{x y} \eta \eta_{A B(i)}-\Sigma P_{y}\left\{\delta_{-A B(Q)}(1-x / l)\right. \\
& \left.-\delta_{R A \in Q}(x / l)\right\}
\end{aligned}
$$

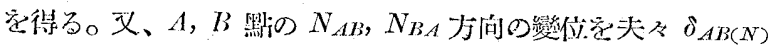
$\delta_{B A(N)}$ とすれば、

$$
\zeta_{A B(i)}=\left(\delta_{A B(N)}+\delta_{B A(N)}\right) / l_{A B}
$$

(5), (7), (8) より、

$$
D_{A B}=\left\{N_{A B(P)}-\Sigma P_{n}(1-x / l)\right\} l_{A B}
$$

なる政、"(10)，(13)，(14)より、

$$
\left.\begin{array}{rl}
D_{A B} \cdot \zeta_{A B(i)} & =N_{A B(P) \delta_{A B(N)}+N_{B A(N)} \delta_{B A(N)}} \\
& -2 P_{x}\left\{\delta_{A B(N)}(1-x / l)-\delta_{B A(N)}(x / l)\right\}
\end{array}\right\}
$$

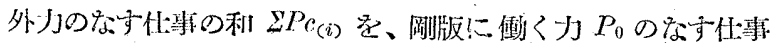

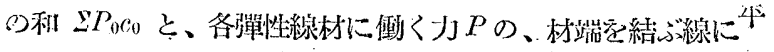

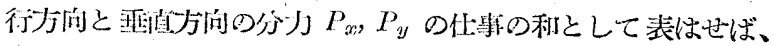

$\Sigma P^{\prime} c_{(i)}=\Sigma P_{0} c_{0}$

$$
\begin{aligned}
+\sum_{A B}[ & -\sum P_{x\left\{\delta_{A B(N)}(1-x / l)-\delta_{B A C(N)}(x / l)\right\}} \\
& +\sum P_{x y \eta_{A B(i)}} \\
& \left.+\sum P_{y}\left\{\delta_{A B(Q)}(1-x / l)-\delta_{P, A(Q)}(x / l)\right\}\right]
\end{aligned}
$$

となる故、(7)，(12)，(15)，(16) より、

$$
\boldsymbol{P}_{i}=\sum_{A}\left\{\sum M_{A}^{0}-\sum_{K} C_{A K}\right\} \xi_{A(i)}+\sum \bar{P}_{(i)}
$$

茲に、 $\sum \bar{P}_{c_{(i)}}=\sum P_{0} c_{0}$

$$
\left.\begin{array}{l}
-\sum_{A B}\left\{Q A B(P) \delta_{A B(Q)}+Q_{B A(Q)} \delta_{B A(Q)}\right. \\
+N_{\left.A B(P) \delta_{A B(N)}+N_{B A(P)} \delta_{B A(N)}\right\}}
\end{array}\right\}
$$

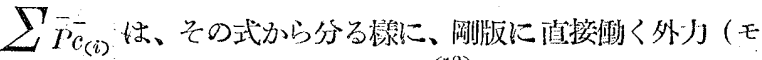

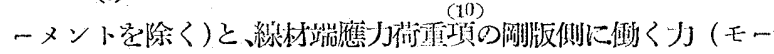

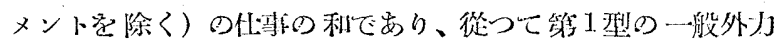

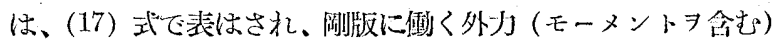
と、紹材端に濑く應力の街重項の䦌版の侧に働くものの總て が、變非狀態 $i$ の祭に謤す㤌哥の租であるとも云へる。

\section{第 2 節 曲線材を含む;剛節骨組に對する 一般化した撓角法}

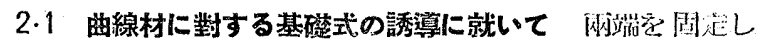

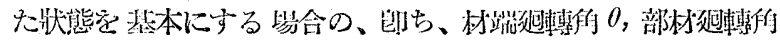

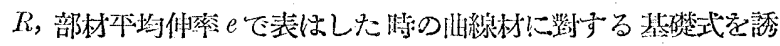
薄与るに際して、若しそれが折總材として报へる埸合には、一

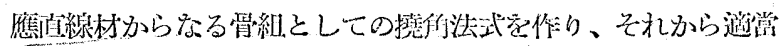

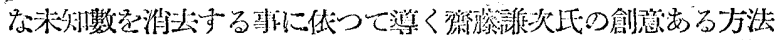

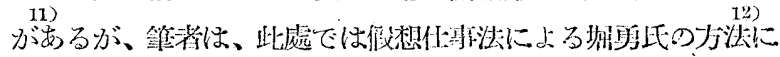
做ひ、をに少し工夫新加一て兄ようと思ら。

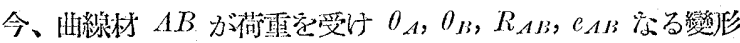

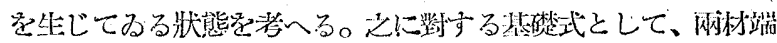
の脂げモーメント $M_{A B}, M_{B A}$ と部材伏長モーメント $T_{A B}$ を

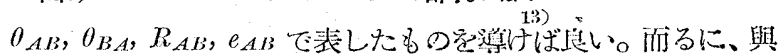

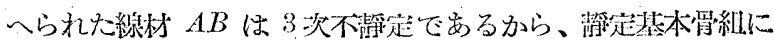

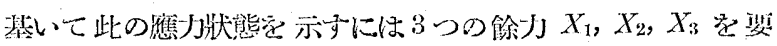
し、任意の應力クは累加法則により次式で泰はされる。 訨台。

$$
Z=Z_{11}-Z_{1} X_{1}-Z_{2} X_{2}-Z_{3} X_{3}
$$

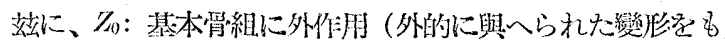
含も）の久方湩いた特の Zの值 $Z_{1}, Z, Z_{3}$ : 基本骨組に外作判なく、 $X_{1}=-1, X_{2}=$

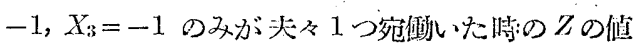
而るに、一方 Zは軤加法則に依り、

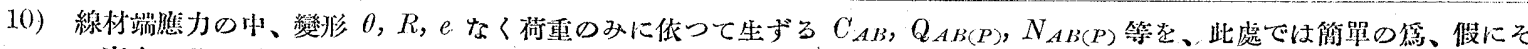

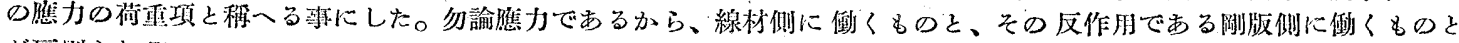
が㗪别さる。

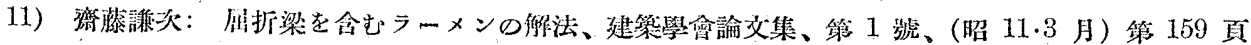
iij I: : 階殺梁を含むラーメンの解法、建䑁學會說文集、第 5 號、(昭 12.3 月) 第 132 頁

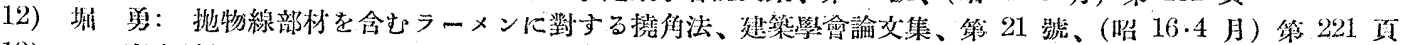

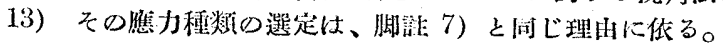


$Z=Z_{\left(D^{2}\right)+}+Z_{(\theta A)} \theta_{A}+Z_{(\theta B)} \theta_{B}+Z_{(L A B)} R_{A B}$ $+T_{(C A B)} e_{A I} \cdots$

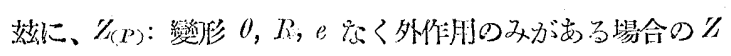

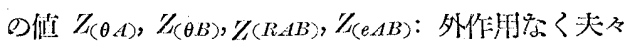
變形 $\theta_{A}=1, \theta_{B}=1, R_{A B}=1, e_{A B}=1$ の 1 つ宛 のみが與へら机た時の $/$ の值

で裴はされるから、(18) 式の $Z_{0}, X_{1}, X_{5}, X_{3}$ に (19) 式家通

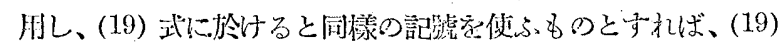

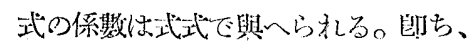

$Z_{(P)}=Z_{1}(P)-Z_{1} X_{1(P)}-Z_{1} X_{(P)}-Z_{3} X_{:(P)}$

$Z_{(\theta A)}=Z_{(0)}(\theta A)-Z_{1} X_{1(\theta A)}-Z_{3} X_{0(\theta A)}-Z_{3} X_{3(\theta A)}$

$Z_{(\theta B)}=Z_{1(\theta)}(\theta B)-Z_{1} X_{1(\theta B)}-Z_{1} X_{:(\theta B)}-Z_{3} X_{3(\theta B)}$

$Z_{(R A B)}=Z_{1(R A B)}-Z_{1} X_{1(R A B)}-Z_{3} X_{(R A B)}-Z_{3} X_{3(R A B)}$

$Z_{(e A B)}=Z_{1(C A B)}-Z_{1} X_{1(e A B)}-Z_{2} X_{2(e A B)}-Z_{3} X_{3(e A B)}$

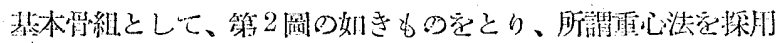

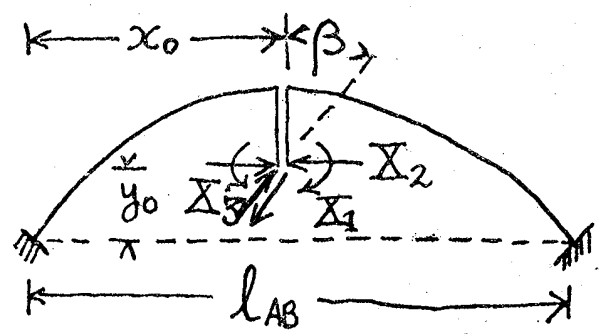

第 2 圖

ナるものとすれば、

$$
Z_{(1)(\theta A)}=Z_{H(1)(\theta B)}=Z_{0(R A B)}=Z_{H}(\dot{e} A B)=0
$$$$
X_{1}=\delta_{10} / \delta_{11}, X_{2}=\delta_{50} / \delta_{20}, X_{3}=\delta_{30} / \delta_{33}
$$

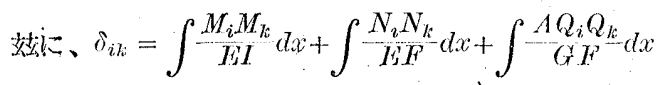

$M_{i}, N_{i}, Q_{i}$ ：外作鹏なく $\mathrm{X}_{i}=-1$ の文が基本骨組

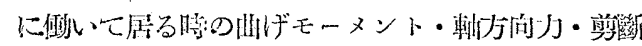

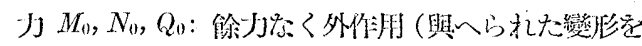

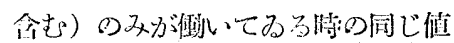

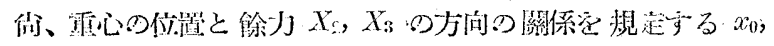

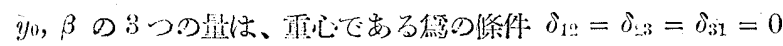

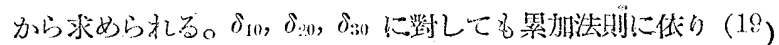

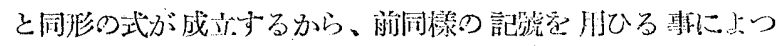
こ、(20), (22) から、

$$
\begin{aligned}
& X_{i(P)}=\delta_{i 0(P)} / \delta_{i i} \\
& X_{i(\theta)}=\delta_{i 0(\theta)} / \delta_{i i} \\
& X_{i(R)}=\delta_{i 0(R)} / \delta_{i i} \\
& X_{i(e)}=\delta_{i *(e) / \delta_{i i}}
\end{aligned}
$$

药に、 $\delta_{i} 0(P)=\int \frac{M_{i} \bar{M}_{0}{ }^{\circ}}{E I} d x+\int \frac{N_{i} \bar{N}_{0}}{H F} d x+\int \frac{\Lambda Q_{i} \bar{Q}_{0}}{G F} d x$

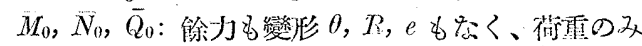

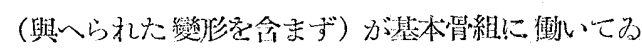

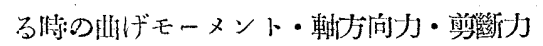

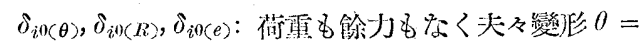
$1, R=1, e=1$ の1つ宛だけ基本骨組に與へた

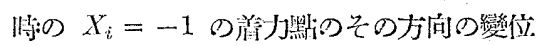

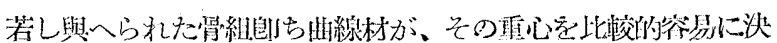

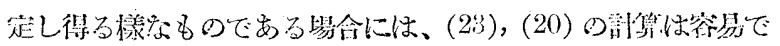

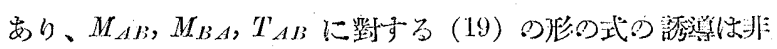

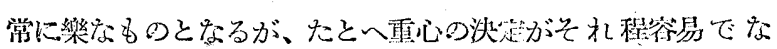
い場合に於ても、上述の方法には、(19) 式の絕龂項（触党、

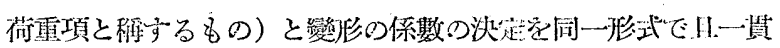
した方法によつてなし得ると去ら特徽が、依然保持されてる bo

仯、(19) 式の變形 $\theta, R, e$ の代りに $\varphi, \psi, \varepsilon$ 芝使子埸命に は、 $0=1, R=1, e=1$ とする代りに $\varphi=1, \psi=1, \varepsilon=1$

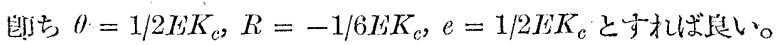

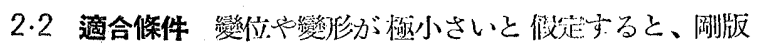

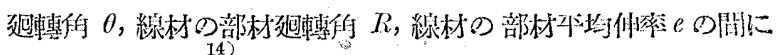

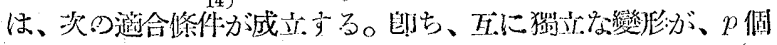
D $\theta, q$ 俔の $R, r$ 俔のeであるとすると、

$$
\left.\begin{array}{rl}
\theta_{i} & =\sum_{j=1}^{p} \xi_{i j}^{\theta} \theta_{j}+\sum_{j=1}^{q} \xi_{i j}^{R} R_{j}+\sum_{j=1}^{r} \xi_{i j}^{e} e_{j} \\
R_{i} & =\sum_{j=1}^{p} \eta_{i j}^{\theta} \theta j+\sum_{j=1}^{q} \eta_{i j}^{R} R j+\sum_{j=1}^{r} \eta_{i j}^{e} e_{i} \\
e_{i} & =\sum_{j=1}^{p} \zeta_{i j}^{\theta} \theta j+\sum_{j=1}^{q} \zeta_{i j}^{R} R j+\sum_{j=1}^{r} \zeta_{i j}^{e} e_{j}
\end{array}\right)
$$

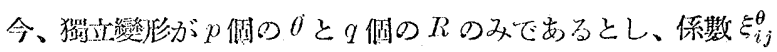

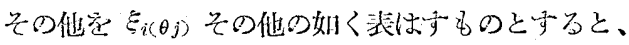

$$
\left.\begin{array}{rl}
\theta_{\imath} & =\sum_{j=1}^{p} \xi_{i(\theta j)} \theta_{j}+\sum_{j=1}^{q} \xi_{i(R j)} R_{j} \\
R_{i} & =\sum_{j=1}^{p} \eta_{i(\theta j)} \theta_{j}+\sum_{j=1}^{q} \eta_{i(R j)} R_{j} \\
e_{i} & =\sum_{j=1}^{p} \zeta_{i(\theta j)} \theta_{j}+\sum_{j=1}^{q} \zeta_{i(R j)} R_{j}
\end{array}\right)
$$

$\theta, R, e$ の代りに、

$$
\begin{aligned}
& \varphi=2 E K_{c} \theta, \phi=-6 E K_{c} R, \varepsilon=2 E K_{c} \theta \\
& \text { 效に、 } F \text { : ヤング係數 }
\end{aligned}
$$

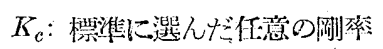

を採朋するものとす机ば、(24) 式は、

$$
\left.\begin{array}{rl}
\varphi_{i} & =\sum_{j=1}^{p} \xi_{i(\theta j)} \varphi_{j}-\frac{1}{3} \sum_{j=1}^{q} \xi_{i(R j)} \phi_{j} \\
\psi_{i} & =-3\left\{\sum_{j=1}^{p} \eta_{i(\theta j)} \varphi_{j}-\frac{1}{3} \sum_{j=1}^{q} \eta_{i(\lambda j)} \psi_{j}\right\} \\
\varepsilon_{i} & =\sum_{j=1}^{p} \zeta_{i(\theta)} \varphi_{j}-\frac{1}{3} \sum_{j=1}^{q} \zeta_{i(R j)} \varphi_{j}
\end{array}\right\} \cdots \cdots \cdots(26)
$$

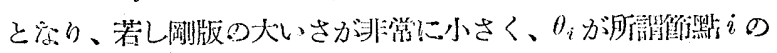

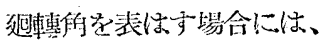

$$
\begin{aligned}
& \xi_{i(\theta i)}=1, \xi_{j(\theta k)}=0(j \neq k), \\
& \xi_{i(R j)}=\eta_{i(\theta j)}=\zeta_{i(\theta j)}=0, \\
& \psi_{i}=\sum_{j=1}^{q} \eta_{i(R j\rangle} \psi_{j},
\end{aligned}
$$

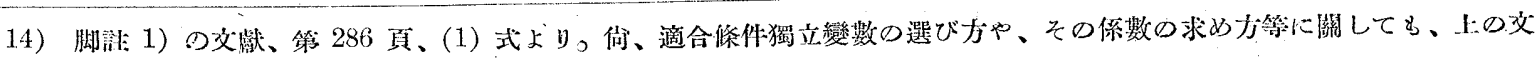
献參照。 


$$
\varepsilon_{i}=-\frac{1}{3} \sum_{j=1}^{q} \zeta_{i(R j)} \psi_{j}
$$

となる。

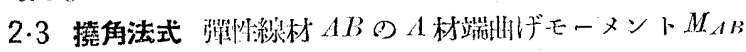

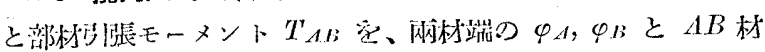
の $\psi_{A B}, \varepsilon_{A B}$ で裴はしたもの定、

$M_{A B}=a_{A B} \varphi_{A}+b_{A B} \varphi_{B}+c_{A B} \psi_{A B}+d_{A B} \varepsilon_{A B}+C_{A B}$
$T_{A B}=a_{A B}^{\prime} \varphi_{A}+b_{A B}^{\prime} \varphi_{B}+c_{A B}^{\prime} \varphi_{A B}+d_{A B} \varepsilon_{A B}+D_{A B}$

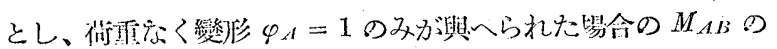

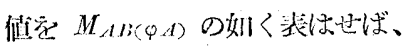

$$
\begin{aligned}
& a_{A B}=M_{A B(\varphi A)}=M_{A B(\theta A)} / 2 E K_{c} \\
& b_{A H}=M_{A B} B(\varphi B)=M_{A B(\theta B)} / 2 H K_{c}=b_{A H} \\
& c_{A A B}=M_{A B(\psi A B)}=-M_{A B(R A B)} / 6 E K_{c} \\
& =\left(a_{A B}+b_{A B}\right) / 3 \\
& d_{A B}=M_{A B(\varepsilon A B)}=M_{A B(e A B)} / 2 E K_{s} \\
& a_{A B}^{\prime}=T_{A B(\varphi, A)}=T_{A B(\theta A)} / 2 E K C \\
& =b^{\prime} B A=d_{A B} \\
& b_{A B}=T_{A B(\varphi B)}=T_{A B(\theta B)} \mid 2 E K_{C} \\
& =a_{B A}^{\prime}=d_{B A A} \\
& c_{A B}^{\prime}=T_{A B}^{\prime}(\Psi A B)=-T_{A B(B A B)}^{\prime} / 6 E K_{C} \\
& \left.=\left(a_{A B}^{\prime}+b_{A B}^{\prime}\right) / 3=c^{\prime}{ }_{B A}=\left(d_{A B}+d_{B A}\right) / 3\right) \\
& d_{A B}=T_{A B(\varepsilon A B)}=T_{A B(C A B)} / 2 E K_{c}
\end{aligned}
$$

なる故、(27) 式は、

$$
\left.\begin{array}{c}
M_{A B}=a_{A B} \varphi_{A}+b_{A B} \varphi_{B}+\left\{\left(a_{A B}+b_{A B}\right) / 3\right\} \psi_{A B} \\
+d_{A B} \varepsilon_{A B}+C_{A B} \\
T_{A B}=d_{A B} \varphi_{A}+d_{B A} \varphi_{B}+\left\{\left(d_{A B}+d_{B A}\right) / 3\right\} \psi_{A B} \\
+d_{A B} \varepsilon_{A B}+D_{A B}
\end{array}\right\}
$$

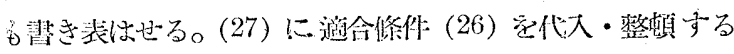
そ.

$$
\left.\begin{array}{l}
M_{A B}=\sum_{j=1}^{p} M_{A B\left(\varphi_{j}\right)} \varphi_{j}+\sum_{j=1}^{q} M_{A B(\psi j)} \psi_{j}+C_{A B} \\
T_{A B}=\sum_{j=1}^{p} T_{A B\left(\varphi_{j}\right)} \varphi_{j}+\sum_{j=1}^{q} T_{A B(\psi j)} \psi_{j}+D_{A B}
\end{array}\right\}
$$

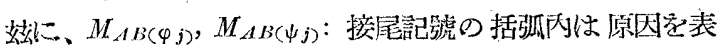
はし、條重なく、夫及摆立變形 $\varphi_{j}=1, \phi_{j}=1 の$ 1 つのみが與へら机壯の $M_{A B}$ の值で烒で表は 也吉。

$$
\begin{aligned}
& M_{A B(\varphi, j)}=a_{A B} \xi_{d(\theta j)}+b_{A B} \xi_{B(\theta, j)}
\end{aligned}
$$

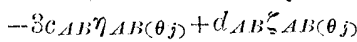

$$
\begin{aligned}
& M_{A B(\psi j)}=-\frac{1}{3}\left\{a_{A B} \xi_{A(R j)}+b_{A B} \xi_{B(R j)}\right. \\
& \left.-3 C_{A B} \eta_{A B(R, j)}+d_{A B} B_{A B(R, j)}\right\}
\end{aligned}
$$

$$
\left.\begin{array}{rl}
T_{A B\left(\varphi_{j}\right)} & =a_{A B}^{\prime} \xi_{A(\theta j)}+b_{A B}^{\prime} \xi_{B(\theta j)} \\
& -3 c_{A B}^{\prime} \eta_{A B(\theta j)}+d_{A B}^{\prime} \xi_{A B(\theta j)} \\
T_{A B(\psi j)} & =-\frac{1}{3}\left\{a_{A B}^{\prime} \xi_{A(R j)}+b_{A B}^{\prime} \xi_{B(R j)}\right. \\
& \left.-3 c_{A B}^{\prime} \eta_{A B(R j)}+d_{A B}^{\prime}{ }_{A B(R j)}\right\}
\end{array}\right\}
$$

となる故、(7) の變形にに街應する一般應力 $\boldsymbol{S}_{i}$ は、

$$
\boldsymbol{S}_{i}=\sum_{j=1}^{p} \boldsymbol{S}_{i\left(\varphi_{j}\right)} \varphi_{j}+\sum_{j=1}^{q} \boldsymbol{S}_{i\left(\psi_{j}\right)} \psi_{j}
$$

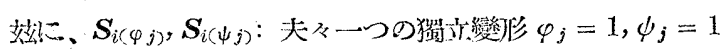

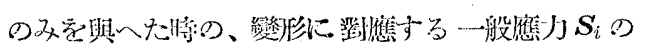
任で、次式で埊はさ机る。

$$
\begin{aligned}
S_{i\left(\varphi_{j}\right)} & =\sum_{A}\left\{\sum_{K} M_{A K\left(\varphi_{j}\right)}\right\} \xi_{A(i)} \\
& -\sum_{A B}\left\{M_{A B\left(\varphi_{j}\right)}+M_{B A\left(\varphi_{j}\right)}\right\} \eta_{A B(i)} \\
& +\sum_{A B} T_{A B(\varphi j)} \sigma_{A B(i)} \\
S_{i(\psi j)} & =\sum_{A}\left\{\sum_{K} M_{A K(\psi j)}\right\} \xi_{A(i)} \\
& -\sum_{A B}\left\{M_{A B(\psi j)}+M_{B A(\psi j)}\right\} \eta_{A B(i)} \\
& \left.+\sum_{A B} T_{A B(\psi j)}\right)_{A B(i)}
\end{aligned}
$$

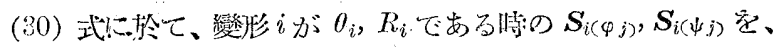
夫及 $\boldsymbol{S}_{\theta i(\varphi j)}, \boldsymbol{S}_{\theta i(\psi j)} ; \boldsymbol{S}_{R i\left(\varphi_{j}\right)}, \boldsymbol{S}_{R i(\psi j)}$ で表はぜぜ、(29)1,

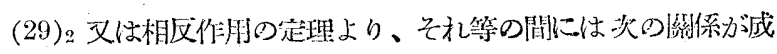
立する事が判る。戕台、

$$
\left.\begin{array}{l}
\boldsymbol{S}_{\theta i\left(\varphi_{j}\right)}=\boldsymbol{S}_{\theta i(\theta j)} / 2 E K_{c}=\boldsymbol{S}_{\theta j(\theta i)} / 2 E K_{c}=\boldsymbol{S}_{\theta j(\varphi i)} \\
\boldsymbol{S}_{R i\left(\varphi_{j}\right)}=\boldsymbol{S}_{R i(\theta j)} / 2 E K_{c}=\boldsymbol{S}_{\theta j(R i)} / 2 E K_{c}=-3 \boldsymbol{S}_{\theta j(\psi i)} \\
\boldsymbol{S}_{R i(\psi j)}=-\boldsymbol{S}_{R i(R j)} / 6 E K_{c}=-\boldsymbol{S}_{R j(R i)} / 6 E K_{c}=\boldsymbol{S}_{R j(\psi i)}
\end{array}\right\}
$$

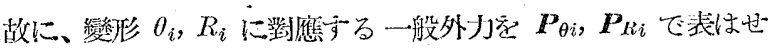
ば、變形 $\theta_{i}, R_{i}$ に對應する力の釣合保件式は、(3)，(30)より、

$$
\left.\begin{array}{l}
\sum_{j=1}^{p} \boldsymbol{S}_{\theta i\left(\varphi_{j}\right)} \boldsymbol{\varphi}_{j}+\sum_{j=1}^{q} \boldsymbol{S}_{\theta i(\psi j)} \psi_{j}=\boldsymbol{P}_{\theta i} \\
\sum_{j=1}^{p} \boldsymbol{S}_{R i\left(\varphi_{j}\right)} \varphi_{j}+\sum_{j=1}^{q} \boldsymbol{S}_{\boldsymbol{R} i\left(\psi_{j}\right)} \psi_{j}=\boldsymbol{P}_{R i}
\end{array}\right\}
$$

又は、(31)より、

$$
\left.\begin{array}{l}
\sum_{j=1}^{p} \boldsymbol{S}_{\theta i(\varphi j)} \boldsymbol{\varphi}_{j}+\sum_{j=1}^{q} \boldsymbol{S}_{\theta i(\psi j)} \psi_{j}=\boldsymbol{P}_{\theta i} \\
\sum_{j=1}^{p} \boldsymbol{S}_{\theta j(\psi i) \varphi_{j}}+\sum_{j=1}^{q}\left(-\boldsymbol{S}_{R i(\psi j)} / 3\right) \psi_{j}=-\boldsymbol{P}_{R i} / 3
\end{array}\right\}
$$

此の (32), (32') が、 $\varphi_{i}(i=1,2, \cdots, p), \phi_{j}(j=1,2, \cdots, q)$ 家 来知数にした時の一般化した萝的法式で嵓り、(32')は、その

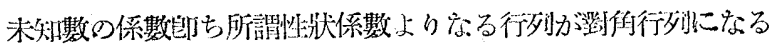
橡に整理されたものでする。

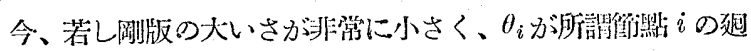

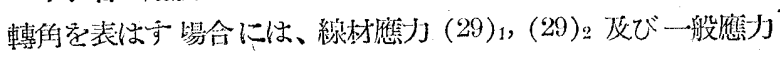
(30) 1 等性次の如くなる。

$$
\left.\begin{array}{l}
M_{A B(\varphi A)}=a_{A B}, M_{A B(\varphi B)}=b_{A B}, M_{A B(\varphi j)}=0(j \neq A, B), \\
M_{A B(\psi j)}=c_{A B} \eta_{A B\left(R_{j}\right)}-d_{A B}{ }_{A B\left(R_{j}\right)} / 3 \\
T_{A B(\varphi A)}=a_{A B}, T_{A B(\varphi B)}=b_{A B}, T_{A B(\varphi j)}=0(j \neq A, B), \\
T_{A B(\psi j)}=c_{A B}^{\prime} \eta_{A B\left(H_{j}\right)}-d_{A B}^{\prime}{ }_{A B(B, j)} / 3
\end{array}\right\}
$$

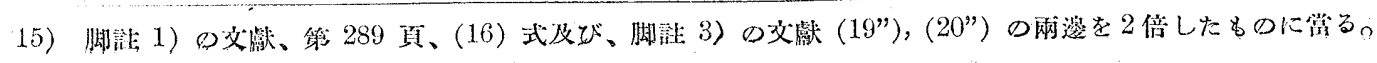




$$
\begin{aligned}
& \boldsymbol{S}_{\theta i(\varphi i)}=\sum_{K} M_{i K(\varphi)}=\sum_{K} a_{i K}, \\
& \boldsymbol{S}_{\theta i(\varphi j)}=M_{i j(\varphi j)}=b_{i j}, \\
& \boldsymbol{S}_{\theta i(\psi j)}=\sum_{K} M_{i K(\psi j)} \\
& \boldsymbol{S}_{L i(\psi, j)}=\sum_{A B}\left[-\left\{M_{A B(\psi j)}+M_{B A(\psi j)}\right\} \eta_{A B(R i)}\right. \\
& \left.+T_{A B(\psi j)} \div A B\left(N_{i}\right)\right]
\end{aligned}
$$

(a)

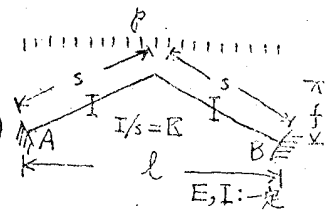

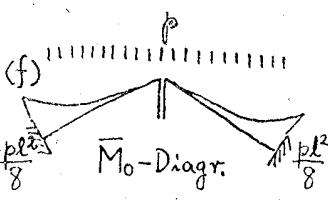

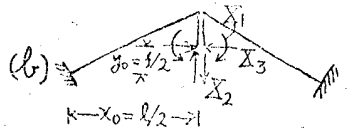

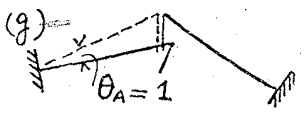

(c)
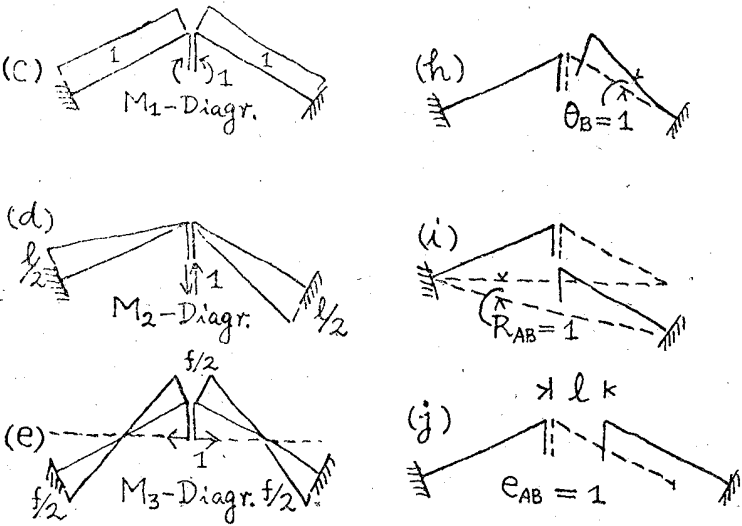

第 3 、圖

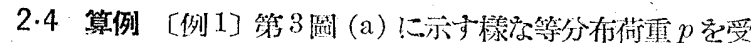

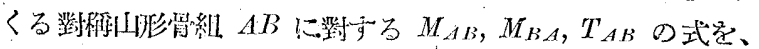
2.1 に於て詨明した方法に依つて求めて見当。(可論今の場合、

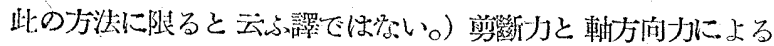

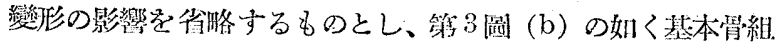

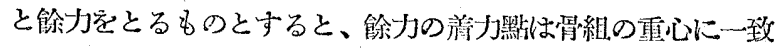
ᄂ. $M_{1^{-}}, M_{2^{-}}, M_{3}$-Diagram は (c), (d), (e)となり、 $\delta_{12}=\delta_{23}=\delta_{31}=0$,

$\delta_{11}=2 / E K, \delta_{22}=l^{2} / 6 E K, \delta_{33}=f^{2} / 6 E K$

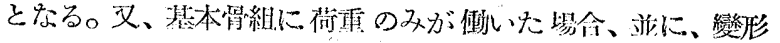
$\theta_{A}=1, \theta_{B}=1, R_{A B}=1, e_{A B}=1$ の夫々 1 つ宛方斯いられ

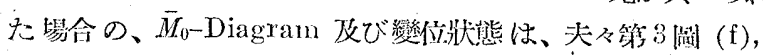
$(\mathrm{g}),(\mathrm{h}),(\mathrm{i}),(\mathrm{j})$ の如くなるから、

$$
\begin{aligned}
& \delta_{10\left(P^{\prime}\right)}=\eta l^{2} / 12 E K . \delta_{00\left(P^{\prime}\right)}=0, \delta_{30\left(P^{\prime}\right)}=-p l^{2} f / 48 \mathrm{EK}, \\
& \delta_{10(\theta A)}=1, \delta_{90(\theta A)}=l / 2, \delta_{30(\theta A)}=-f / 2, \\
& \delta_{10(\theta B)}=-1, \delta_{\Omega 0(\theta B)}=l / 2, \delta_{30(\theta / B)}=f / 2 \text {, } \\
& \delta_{10(R A B)}=0, \delta_{0(R A B,}=-l, \delta_{30(R A B)}=0 \text {, } \\
& \delta_{10(e A B)}=0, \delta_{0(0) A B)}=0, \delta_{3 v(C A B)}=l,
\end{aligned}
$$

故に、(23) 式に依り、

$X_{1\left(l^{\prime}\right)}=p l^{2} / 24, X_{2\left(l^{\prime}\right)}=0, X_{3\left(l^{\prime}\right)}=-p t^{2} / 8 f$,

$X_{1(\theta A)}=E K / 2, X_{2(\theta A)}=3 E K / l, X_{3(\theta A)}=-3 E K / f$,

$X_{1(\theta B)}=-E \stackrel{K}{K} / 2, X_{\therefore(\theta B)}=3 K K / l, X_{3(\theta B)}=3 E K / f$,

$X_{1(R A B)}=0, X_{2(R A B)}=-6 E K / l, X_{3(R A B)}=0$,

$X_{1(e A A)}=0, X_{2(e A B)}=0, X_{3(e A B)}=6 E K l \mid f^{2}$

$(20),(28)$ より、(27) 式の係數は次の如くなる。四ち、

$$
\begin{aligned}
& a_{A B}=7 k / 4, b_{A B}=-k / 4, c_{A B}=k / 2, \\
& d_{A B}=-1 \cdot 5 k l \mid f, C_{A B}=-p l^{2} / 48, \\
& a_{B A}=7 k / 4, b_{B A}=-k / 4, c_{B A}=k / 2, \\
& d_{B A}=1 \cdot 5 k l \mid f, C_{B A}=p l^{2} / 48, \\
& a_{A B}^{\prime}=-1.5 k l\left|f, b_{A B}^{\prime}=1 \cdot 5 k l\right| f, c_{A B}^{\prime}=0, \\
& d_{A B}^{\prime}=3 k l^{2} \mid f^{2}, D_{A B}=-p l^{\prime \prime} / 8 f
\end{aligned}
$$

\begin{tabular}{|c|c|c|c|c|c|c|c|c|c|c|}
\hline & $a$ & $b$ & $c$ & $d$ & $\sigma$ & $a^{\prime}$ & $b^{\prime}$ & $c^{\prime}$ & $d^{\prime \prime}$ & $D$ \\
\hline $\begin{array}{l}A \\
B\end{array}$ & $\begin{array}{l}2 \\
2\end{array}$ & 1 & 1 & $\begin{array}{l}0 \\
0\end{array}$ & $\begin{array}{l}0 \\
0\end{array}$ & 二 & - & - & - & - \\
\hline$B$ & $\begin{array}{l}2.1 \\
2.1 \\
\end{array}$ & $-0 \cdot 3$ & 0.6 & $\begin{array}{r}7 \\
-7 \cdot 2 l / h \\
7 \cdot 2 l / h \\
\end{array}$ & $\begin{array}{r}-w l^{2} / 12 \\
w l^{2} / 12\end{array}$ & $\begin{array}{r}-7 \cdot 2 l / h \\
7.2 l / h\end{array}$ & $\begin{array}{r}7.27 / h \\
-7.27 / h \\
\end{array}$ & 0 & $57 \cdot 6(l / h)^{2}$ & $-2 w l^{3} / h$ \\
\hline $\begin{array}{l}D \\
L\end{array}$ & $\begin{array}{l}2 \\
2\end{array}$ & 1 & 1 & $\begin{array}{l}0 \\
0\end{array}$ & $\begin{array}{l}0 \\
0\end{array}$ & - & - & - & - & - \\
\hline$H$ & $\begin{array}{l}4.8 \\
4.8 \\
\end{array}$ & $2 \cdot 4$ & $2 \cdot 4$ & $\begin{array}{l}0 \\
0\end{array}$ & $\begin{array}{r}-w l^{2} / 12 \\
w l^{2} / 12\end{array}$ & - & - & - & - & - \\
\hline Ir & 4 & 2 & 2 & $\begin{array}{l}0 \\
0\end{array}$ & $\begin{array}{l}0 \\
0\end{array}$ & E & $=$ & - & - & - \\
\hline
\end{tabular}

\section{$w$}

\section{1}

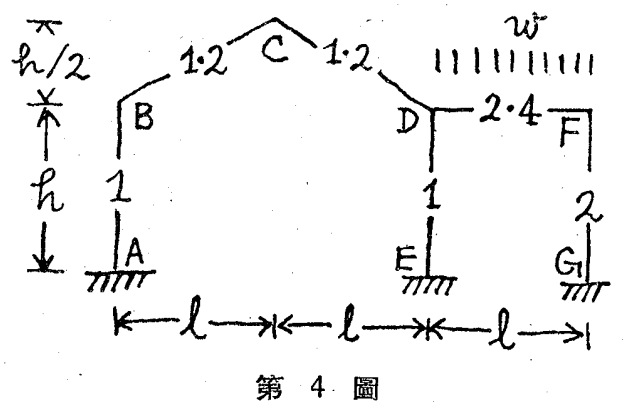

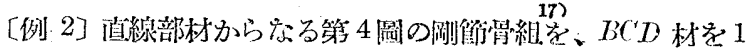
部材と考へて撓的法に依つて解いて其る。但し、材料は一定、

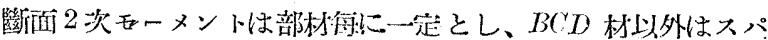
ンの長さの變化なしと考へるものとする。今、 $A B, B D, D L$, $D F, F G$ 材に對する (27) 式の係數を數表の形に整頓して表 亦すると、(例 1 の結果 (33) 式參照) 炏の如くなる。

16）炎の文墑に掍げられて子る公式に一致する。

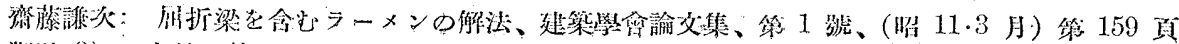

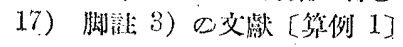


今、 $A B, D E$ 基本部材に選び、 $\eta, \zeta, M_{(\psi A B)}, M_{(\psi D E)}, T_{(\psi A B)}, T_{(\psi D E)}$ を逐次求め、

$S_{R i(\psi j)}^{p q}=-\left\{M_{p q(\psi j)}+M_{q p(\psi j)}\right\} \eta_{p q(R i)}+T_{p q(\psi j)} \zeta_{p q(R i)}$

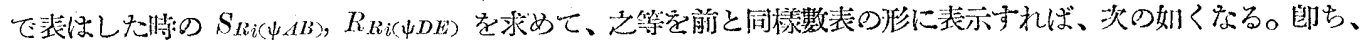

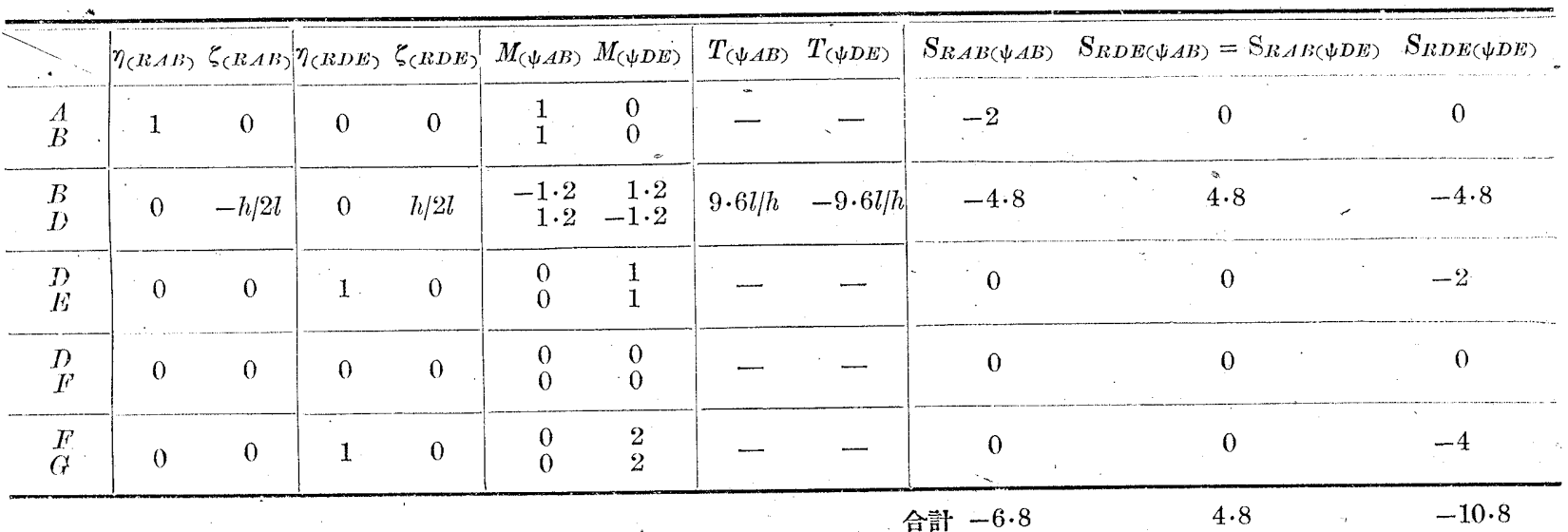

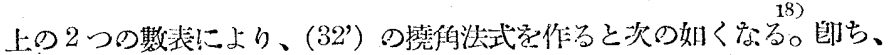

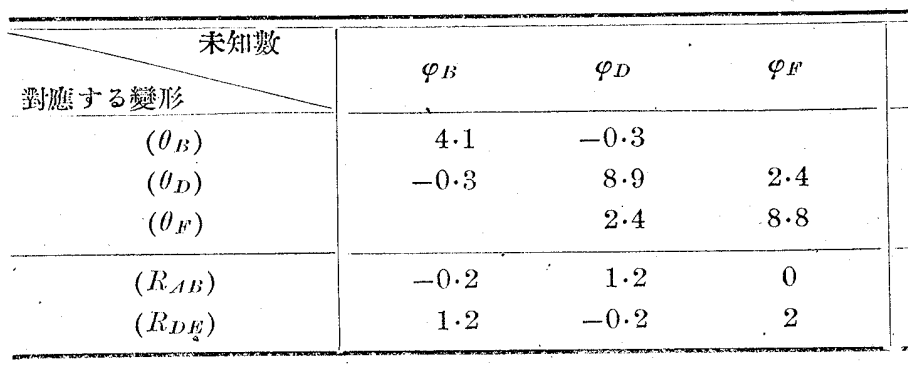

此の方程式客朷けば、(單位:

$\varphi_{B}=0.50963, \varphi_{D}=-0.21972, \varphi_{H}=0.11161$,

$\psi_{A B}=1.41253, \psi_{D E}=-0.72740$

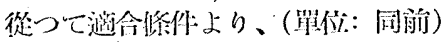

$\phi_{I B D}=\phi_{D H^{*}}=0, \phi_{H^{\prime}} \alpha=-0.72740, \varepsilon_{B D}=0.35666 \mathrm{hll}$

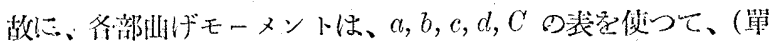
位: $\left.w l^{2} / 12\right)$

$M_{A B}=1.9222, M_{B A}=2.4318$,

\begin{tabular}{cc|c}
\hline$\phi_{A B}$ & $\psi_{D E}$ & 嗬重项（單位: $\left.u l^{2} / 12\right)$ \\
\hline-0.2 & 1.2 & 1 \\
1.2 & -0.2 & 0 \\
0 & 2 & -1 \\
\hline $6 \cdot 8 / 3$ & -1.6 & 4 \\
-1.6 & 3.6 & -4 \\
\hline
\end{tabular}

$M_{B D}=-2 \cdot 4318, \quad M_{D B}=2 \cdot 9537$

$M_{D E}=-1.1668, \quad M_{E D}=-0.9471$,

$M_{D F^{\prime}}=-1.7868, \quad M_{H^{\prime} D}=1.0084$,

$M_{F^{\prime} G}=-1.0084, \quad M_{G F^{\prime}}=-1.2316$

$T_{H D}$ 定求めると、

$T_{B D D}=-8.7077\left(w l^{*} / 12 h\right)$

$\therefore N_{B D}=-1 \cdot 4513 w l^{2} / h$

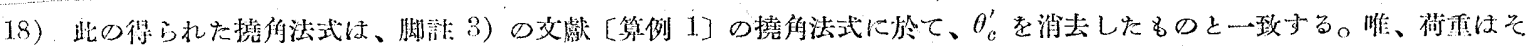

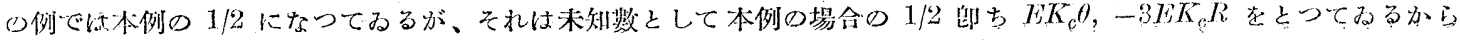
である。算川した次の未知數值を見てもそれが分らら。 